\title{
The value of lymphocyte-to-monocyte
} ratio and neutrophil-to-lymphocyte ratio in differentiating pneumonia from upper respiratory tract infection (URTI) in children: a cross-sectional study

Jinghua Wu ${ }^{1,2+}, X$ Wang $^{3,4+}$, Mingqi Zhou ${ }^{1,2+}$, Guo-Bo Chen ${ }^{5,6}$, Jing Du ${ }^{6}$, Ying Wang ${ }^{6 *}$ and Chengyin Ye $e^{1,2^{*}}$

\begin{abstract}
Backgrounds: Early and accurate diagnosis of pediatric pneumonia in primary health care can reduce the chance of long-term respiratory diseases, related hospitalizations and mortality while lowering medical costs. The aim of this study was to assess the value of blood biomarkers, clinical symptoms and their combination in assisting discrimination of pneumonia from upper respiratory tract infection (URTI) in children.

Methods: Both univariate and multivariate logistic regressions were used to build the pneumonia screening model based on a retrospective cohort, comprised of 5211 children (age $\leq 18$ years). The electronic health records of the patients, who had inpatient admission or outpatient visits between February 15, 2012 to September 30, 2018, were extracted from the hospital information system of Zhejiang Provincial People's Hospital, Hangzhou, Zhejiang Province, China. The children who were diagnosed with pneumonia and URTI were enrolled and their clinical features and levels of blood biomarkers were compared. Using the area under the ROC curve, both two screening models were evaluated under $80 \%$ (training) versus $20 \%$ (test) cross-validation data split for their accuracy.

Results: In the retrospective cohort, 2548 of 5211 children were diagnosed with the defined pneumonia. The univariate screening model reached predicted AUCs of 0.76 for lymphocyte/monocyte ratio (LMR) and 0.71 for neutrophil/ lymphocyte ratio (NLR) when identified overall pneumonia from URTI, attaining the best performance among the biomarker candidates. In subgroup analysis, LMR and NLR attained AUCs of 0.80 and 0.86 to differentiate viral pneumonia from URTI, and AUCs of 0.77 and 0.71 to discriminate bacterial pneumonia from URTI respectively. After integrating LMR and NLR with three clinical symptoms of fever, cough and rhinorrhea, the multivariate screening model obtained increased predictive values, reaching validated AUCs of $0.84,0.95$ and 0.86 for distinguishing pneumonia, viral pneumonia and bacterial pneumonia from URTI respectively.
\end{abstract}

\footnotetext{
*Correspondence: nancywangying@163.com; yechengyin@hznu.edu.cn

${ }^{\dagger}$ Jinghua Wu, Xu Wang and Mingqi Zhou contributed equally to this work.

1 Department of health management, Hangzhou Normal University,

Zhejiang 310000, Hangzhou, China

${ }^{6}$ Phase I Clinical Research Center, Zhejiang Provincial People's Hospital,

Affiliated People's Hospital, Hangzhou Medical College, Zhejiang 310000

Hangzhou, China

Full list of author information is available at the end of the article
}

(C) The Author(s) 2021. Open Access This article is licensed under a Creative Commons Attribution 4.0 International License, which permits use, sharing, adaptation, distribution and reproduction in any medium or format, as long as you give appropriate credit to the original author(s) and the source, provide a link to the Creative Commons licence, and indicate if changes were made. The images or other third party material in this article are included in the article's Creative Commons licence, unless indicated otherwise in a credit line to the material. If material is not included in the article's Creative Commons licence and your intended use is not permitted by statutory regulation or exceeds the permitted use, you will need to obtain permission directly from the copyright holder. To view a copy of this licence, visit http://creativecommons.org/licenses/by/4.0/. The Creative Commons Public Domain Dedication waiver (http://creativeco mmons.org/publicdomain/zero/1.0/) applies to the data made available in this article, unless otherwise stated in a credit line to the data. 
Conclusions: Our study demonstrated that combining LMR and NLR with critical clinical characteristics reached promising accuracy in differentiating pneumonia from URTI, thus could be considered as a useful screening tool to assist the diagnosis of pneumonia, in particular, in community healthcare centers. Further researches could be conducted to evaluate the model's clinical utility and cost-effectiveness in primary care scenarios to facilitate pneumonia diagnosis, especially in rural settings.

Keywords: Pneumonia, Upper respiratory tract infection, Neutrophil-to-lymphocyte ratio, Lymphocyte-to-monocyte ratio

\section{Introduction}

Pneumonia is the leading cause of hospitalizations and death among children globally. In 2015, the estimated cause-specific mortality rate of pneumonia was 5.455 cases per 1000 live births [1]. Therefore, childhood pneumonia causes a significant burden on both patients and their families, including substantial expenses, loss of routine, and decrease in quality of life [2]. Furthermore, pneumonia in early childhood has increasingly been associated with reduced lung function and the development of chronic non-communicable respiratory diseases, such as asthma or chronic obstructive pulmonary disease, both in children and adults [3-6].

Although chest X-ray is considered as a critical step for pneumonia diagnosis with high accuracy, it still has some shortcomings, including high expense, unnecessary check and inconsistency in radiographies by physicians [7]. Accurate diagnosis of pneumonia in primary care remains difficult as it is impractical to send all children to chest X-rays examinations, most primary physicians therefore initially rely on clinical signs and blood routine test [8]. However, due to some overlaps of symptoms between pneumonia and upper respiratory tract infection (URTI), such as fever and cough, it is still a challenge to identify children infected by pneumonia from the patients with URTI, especially for those primary healthcare workers who lack of expertise in diagnosing suspected pneumonia cases or in some resource-poor primary care settings where chest X-ray is unavailable $[9$, 10].

As one of the most practical ways to track patients' physical condition, blood routine test usually measures the levels of neutrophils cell (NC), monocytes cell (MC), lymphocyte cell (LC), white blood cell count (WBC) and C-reactive protein (CRP) in blood. Among them, neutrophils, lymphocytes and monocytes are common indicators of human body's inflammation and immune status. Microbes, such as respiratory syncytial virus, influenza virus, pneumococcus, or staphylococcus aureus, were generally detected in patients with pneumonia [11-13], and the rapid accumulation of neutrophils is recognized as the key to effectively clean up microbe threats [14]. Other leukocytes, including monocytes and lymphoid cells, could also be recruited in such antimicrobial immune process [15]. However, in addition to pneumonia, the levels of these biomarkers may also be affected by other factors, such as leukemia, acute infection and tumor, showing great variations among individuals [1618]. Nevertheless, the calculated neutrophil/lymphocyte ratio (NLR) and lymphocyte/monocyte ratio (LMR) can eliminate such variation and be sensitive to reflect the balance between inflammatory response and immune status in patients [19]. Studies have shown that NLR and LMR are good indicators in evaluating prognosis of various diseases, such as malignant tumors, etc. [20]. With the capacity of notifying inflammatory response and immune status in patients, we wonder whether NLR or LMR could be used as preliminary indicators to screen children at high risk of pneumonia and to help determine the needs for further chest X-ray examinations, as well as to identify children at low risk of pneumonia and avoid unnecessary chest X-ray checks for these low-risk children.

In this study, we aimed to develop a clinical primary screening tool to differentiate pneumonia from URTI, by using children's EHR data in Zhejiang Provincial People's Hospital, Hangzhou, Zhejiang Province, China. We anticipate the tool can help clinicians make clinical decisions about who should be sent for chest radiography examination to identify possible pneumonia, and facilitate precise diagnosis by reducing unnecessary medical expenses.

\section{Methods}

\section{Cohort}

According to the corresponding inclusion and exclusion criteria described below, a total of 5211 eligible patients that have inpatient admission or outpatient visits to Zhejiang Provincial People's Hospital from February 15, 2012 to September 30, 2018, were enrolled in this retrospective study cohort. In this retrospective observational study, the use of the de-identified data was authorized, patients having diagnostic records of URTI, viral or bacterial pneumonia, were extracted from the EMR big data intelligent platform of Zhejiang Provincial People's Hospital information system, along with their demographics, laboratory test results, chest $\mathrm{x}$-ray records, and clinical 
symptoms information at the time of their initial inpatient admission or outpatient visits. All personal privacy information was well protected and removed during the analysis and publication process. This study was approved by ethics committee of Zhejiang Provincial People's Hospital (No. 2021QT222), and was exempt from informed consent as shown by the IRB approval letter. Since this was a diagnostic accuracy study, we followed the Standards for Reporting of Diagnostic Accuracy Studies (STARD) and completed the STARD checklist (see Supplementary Table 1) [21].

The inclusion and exclusion criteria were demonstrated carefully in the study design workflow (Fig. 1), and the detailed diagnostic criteria of URTI and pneumonia were summarized in Supplementary Table 2. Specifically, patients with URTI were defined as those who meet all of the following criteria: 1) under the age of 18 years, 2) having clinical signs such as cough, swollen and congested tonsils, hyperemia, edema, and secretions in nasal mucosa or pharynx, or runny nose, body temperature $>37^{\circ} \mathrm{C}$, and no abnormalities in lung auscultation, 3) having normal or low WBC and increased lymphocyte ratio that indicates viral infections, or increased WBC and neutrophils ratio that implies bacterial infections, 4) showing no pulmonary imaging changes in chest X-ray if any. Those who had received antibiotics before hospital visits or were diagnosed with pneumonia, bronchitis, and other lower respiratory tract infections at hospital were excluded from the URTI cohort. Patients with viral pneumonia were defined as children who meet all of the following criteria during their hospital visits: 1 ) under the age of 18 years, 2) having clinical symptoms of cough, body temperature between $37^{\circ} \mathrm{C}$ and $38.5^{\circ} \mathrm{C}$ generally, wheezing in auscultation, tachypnoea, breathlessness, or chest pain, etc., 3) having normal or low WBC and increased lymphocyte ratio, 4) showing multifocal 1-10$\mathrm{mm}$ well-defined or ill-defined nodular opacity with a surrounding halo or patchy ground-glass opacity (GGO), or other clues of viral pneumonia in the chest radiograph, as summarized in Supplementary Table 2, 5) having any of 8 types of respiratory viruses detected from nasopharyngeal or throat swabs, including respiratory syncytial virus (RSV), adenovirus nucleic acid (ADVDNA), influenza A virus antigen (FluA-Ag), and so on. Patients diagnosed with pneumonia caused by bacteria or other pathogens were excluded from the viral-pneumonia cases. Patients with bacterial pneumonia were defined as those meet all of the following criteria: 1) under the age of 18 years, 2) having clinical symptoms of cough, body temperature $\geq 38.5^{\circ} \mathrm{C}$ generally, rhonchus or moist rales in lung auscultation, tachypnoea, breathlessness, or chest pain, etc., 3) having increased WBC and neutrophils

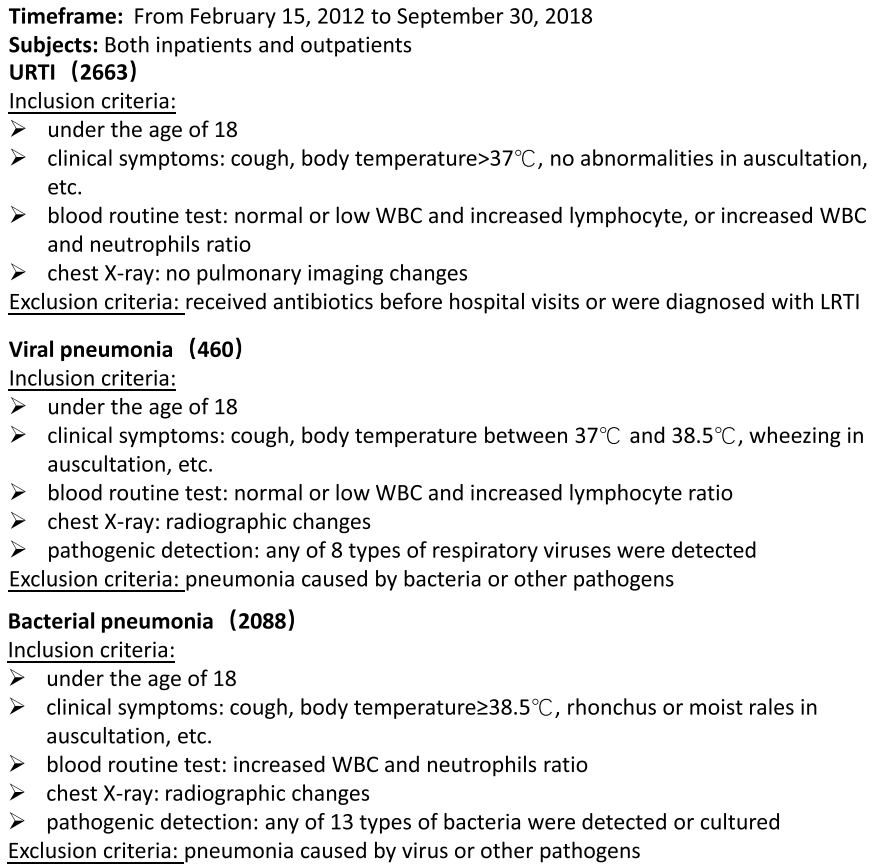

blood routine test: normal or low WBC and increased lymphocyte, or increased WBC and neutrophils ratio

$>$ chest X-ray: no pulmonary imaging changes

Exclusion criteria: received antibiotics before hospital visits or were diagnosed with LRTI

Viral pneumonia (460)

Inclusion criteria:

$>$ under the age of 18

clinical symptoms: cough, body temperature between $37^{\circ} \mathrm{C}$ and $38.5^{\circ} \mathrm{C}$, wheezing in auscultation, etc.

$>$ blood routine test: normal or low WBC and increased lymphocyte ratio

$>$ chest $X$-ray: radiographic changes

$>$ pathogenic detection: any of 8 types of respiratory viruses were detected Exclusion criteria: pneumonia caused by bacteria or other pathogens

Bacterial pneumonia (2088)

Inclusion criteria:

$>$ under the age of 18

$>$ clinical symptoms: cough, body temperature $\geq 38.5^{\circ} \mathrm{C}$, rhonchus or moist rales in auscultation, etc.

$>$ blood routine test: increased WBC and neutrophils ratio

$>$ chest $\mathrm{X}$-ray: radiographic changes

$>$ pathogenic detection: any of 13 types of bacteria were detected or cultured Exclusion criteria: pneumonia caused by virus or other pathogens

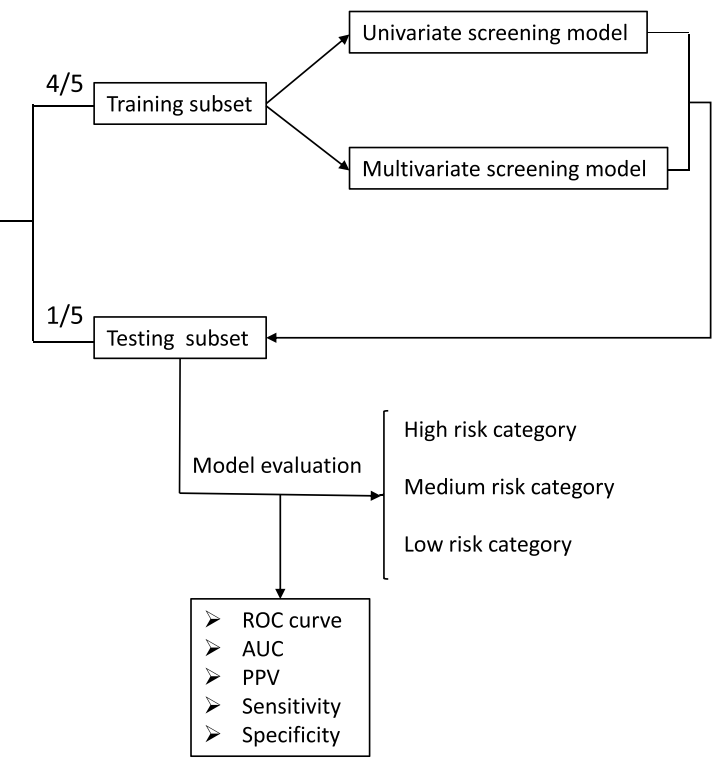

Fig. 1 Study design 
ratio, 4) showing patchy shadow, lung consolidation (usually lobed or segmentary with bronchial inflation), centrilobular lung nodules (Solid or mixed density nodules along the bronchovascular bundle), or other signs of bacterial pneumonia in the chest radiograph, as summarized in Supplementary Table 2, 5) having any of 13 types of bacteria detected or cultured from nasopharyngeal swabs, sputum, alveolar lavage fluid, pleural effusion or fiberoptic bronchoscopy smear, such as pseudomonas aeruginosa, candida albicans, mixed flora, klebsiella pneumoniae, and so on. Those who diagnosed with pneumonia caused by virus or other pathogens were excluded from the bacterial-pneumonia cases. Since the data of this retrospective cohort study was extracted from the big data platform, only those having complete diagnostic information, clinical symptoms or signs, blood routine examination and having chest $\mathrm{X}$-ray results when diagnosed with pneumonia were included for analysis.

\section{Specimen collection and laboratory procedures}

As a routine blood test for children presenting to the facility, the complete blood count was conducted on each patient to measure the concentration of neutrophils cell (NC), monocytes cell (MC), lymphocyte cell (LC), white blood cell count (WBC), C-reactive protein (CRP). Pleural effusion puncture was performed under ultrasound guidance so as to extract pleural effusion from chest cavity. Microscopes, culture medium or polymerase chain reaction (PCR) were used to detect pathogenic bacteria. In accordance to the standard sample collection procedures, nasopharyngeal or throat swabs were collected to detect respiratory viruses or bacteria. Sputum was cultured for anaerobes, aerobes, fungus, as well as mycobacterium tuberculosis, in order to diagnose the causes of respiratory infection. To obtain fiberoptic bronchoscopy smear, the fiberoptic bronchoscopy was brushed twice at the suspicious site under tracheal mirror positioning and the entrapped cells were smeared and sent for examination. The alveolar bronchus was repeatedly rinsed with normal saline to obtain alveolar lavage fluid. The fluid was further centrifuged at $2000 \mathrm{r} / \mathrm{min}$ for $3 \mathrm{~min}$, where the supernatant was discarded and the sediment was smeared.

\section{Statistical analysis}

Categorical variables were compared using chis-square and continuous variables were assessed using MannWhitney $U$ test. The sample.split function from package caTools of R libraries was applied to split the study cohort randomly into two subsets at the predefined ratio. In our study, the retrospective cohort (5211 patients) was split into the training and testing subsets at a ratio of $4: 1$, where the number of pneumonia and URTI cases was uniformly distributed across the training and testing subsets. The modeling process was implemented in two phases: (1) the training subset was used to develop the model and generate predictive estimates and (2) then was validated in the testing subset. First, we built the univariate screening model by treating blood biomarkers (WBC, NC, MC, LC, CRP, LMR, NLR) independently as discriminators. Among them, NLR was calculated as the ratio of neutrophils to lymphocytes while LMR was calculated as the ratio of lymphocytes to monocytes. Secondly, we built the multivariate screening model by combining symptoms of age, fever, cough and rhinorrhea (RHI), and the most significant biomarkers (NLR and LMR) all together as pneumonia indicators. For both univariate and multivariate model, each individual's risk score in either training or validation cohort was calculated firstly. Then, individuals in training set were sorted by their risk scores from low to high, and the 25 and $75 \%$ quantiles were obtained as cut-offs to divide individuals into three risk categories. After that, these determined cut-offs were applied to the validation cohort, thereafter, three risk categories of the validation set were captured, indicating the high, medium, and low risks of pneumonia. Following that, positive predictive values (PPVs), sensitivity and specificity were carefully calculated. A subgroup analysis was also performed to evaluate the above built models for distinguishing two different types (bacterial and viral) of pneumonia from URTI. The area under the ROC curve was used to evaluate the accuracy of the screening model. All statistical analyses were conducted in $\mathrm{R}$ software version 3.6.1. R libraries, including glm, ROCR, AUC, ggplot and $\mathrm{pROC}$, were applied respectively.

\section{Results}

Baseline characteristics

A total of 5211 children were included in our study. Among them, 2548 (48.9\%) were diagnosed with pneumonia and 2663 (51.1\%) were with URTI. Baseline demographic features, clinical features, as well as the blood inflammatory markers, were summarized in Table 1. Age showed statistical difference between the two sub-cohorts $(p<0.05)$, the overall distribution of children with pneumonia was balanced in all age groups, while the number of children with URTI generally increases with age, with only $9.76 \%$ of URTI population younger than 1-year-old and $43.94 \%$ older than 7 years of age. Gender showed no difference between the two sub-cohorts $(p>0.05)$, with the male patients occupying $54.3 \%$ of pneumonia and $53.44 \%$ of URTI patients respectively. Three major clinical symptoms showed statistical difference between the two sub-cohorts, where the frequency of fever (defined as temperature $\geq 37.0^{\circ} \mathrm{C}$ ) and coughing was higher in patients with pneumonia, while more rhinorrhea individuals were 
Table 1 Distribution of baseline features in children diagnosed with pneumonia and URTI

\begin{tabular}{|c|c|c|}
\hline Parameter & $\begin{array}{l}\text { Pneumonia } \\
(N=2548)\end{array}$ & $\begin{array}{l}\text { URTI } \\
(N=2663)\end{array}$ \\
\hline \multicolumn{3}{|l|}{ Demographic, n (\%) } \\
\hline \multicolumn{3}{|l|}{ Age (years)* } \\
\hline$<1$ & $741(29.08)$ & $260(9.76)$ \\
\hline $1-2$ & $528(20.72)$ & $434(16.3)$ \\
\hline $3-4$ & $484(19)$ & $443(16.64)$ \\
\hline $5-6$ & $310(12.17)$ & $356(13.37)$ \\
\hline$\geq 7$ & $485(19.03)$ & $1170(43.94)$ \\
\hline Male sex & $1384(54.32)$ & $1423(53.44)$ \\
\hline \multicolumn{3}{|l|}{ Clinical features, n (\%) } \\
\hline Fever $\left(\mathrm{BT} \geq 37^{\circ} \mathrm{C}\right) *$ & $1967(77.2)$ & $1705(64.03)$ \\
\hline Cough * & $2365(92.82)$ & $1594(59.86)$ \\
\hline Rhinorrhea* & $561(22.02)$ & $859(32.26)$ \\
\hline \multicolumn{3}{|c|}{ Complete blood count, median (IQR) } \\
\hline WBC $(\times 10 \wedge 9 / L)$ & $7.66(5.91,10.06)$ & $7.78(5.87,10.22)$ \\
\hline $\mathrm{NC}\left(\times 10^{\wedge} 9 / \mathrm{L}\right) *$ & $3.03(1.92,4.63)$ & $4.44(2.83,6.7)$ \\
\hline$M C(\times 10 \wedge 9 / L) *$ & $0.49(0.34,0.71)$ & $0.6(0.45,0.78)$ \\
\hline $\mathrm{EC}\left(\times 10^{\wedge} 9 / \mathrm{L}\right) *$ & $0.14(0.05,0.27)$ & $0.07(0.02,0.18)$ \\
\hline $\mathrm{LC}\left(\times 10^{\wedge} 9 / \mathrm{L}\right) *$ & $3.4(2.22,5.11)$ & $2.07(1.34,3.19)$ \\
\hline $\operatorname{CRP}(\mathrm{mg} / \mathrm{L}) *$ & $3.8(1.2,9.6)$ & $4(1.5,9.8)$ \\
\hline $\mathrm{LMR}^{*}$ & $7.09(4.57,10.61)$ & $3.45(2.14,5.61)$ \\
\hline$N L R^{*}$ & $0.87(0.43,1.76)$ & $2.18(1.09,4.27)$ \\
\hline
\end{tabular}

For the comparison between pneumonia and UTRI, Chi-square test for categorical variables and Wilcoxon-rank sum test for continuous variable was employed; ${ }^{*} p<0.05$ observed in URTI sub-cohort. Most blood biomarkers, except WBC, all showed statistical difference between the two sub-cohorts $(p<0.05)$.

\section{Model performance}

Initially, we divided the data into training and testing sets at a ratio of $4: 1$ to build the univariate screening model, and evaluated the model's performance on an independent testing data. In summary, LMR and NLR showed better validated discriminative accuracy than other biomarkers (LC, NC, MC, WBC and CRP), either in distinguishing the overall pneumonia from URTI or in discriminating subgroups of viral or bacterial pneumonia from URTI. LMR attained the highest accuracy among all biomarkers mentioned above, reaching a predicted AUC of 0.76 (95\% CI: $0.73-0.79)$ on the validation set to distinguish the overall pneumonia from URTI (Fig. 2). NLR also achieved a relatively high validated AUC of 0.71 (95\% CI: 0.68-0.74). On the contrary, while introducing other blood biomarkers into the validation procedure, they all achieved relatively low AUC values (range 0.49-0.69), showing little discriminative values (see Supplementary Table 3). More details about the validated AUCs of each blood biomarkers and their 95\% confidence intervals were shown below (Fig. 3). In the subgroup analysis to distinguish two different types (viral or bacterial) of pneumonia and URTI, the two indicators, LMR and NLR, also demonstrated their promising performance better than all other biomarkers, as they attained predicted AUCs of 0.86 (95\% CI: 0.82-0.90) and 0.80 (95\% CI: 0.76-0.84) in distinguishing viral pneumonia from URTI respectively, and achieved the AUCs of 0.77 (95\%

\section{Pneumonia}

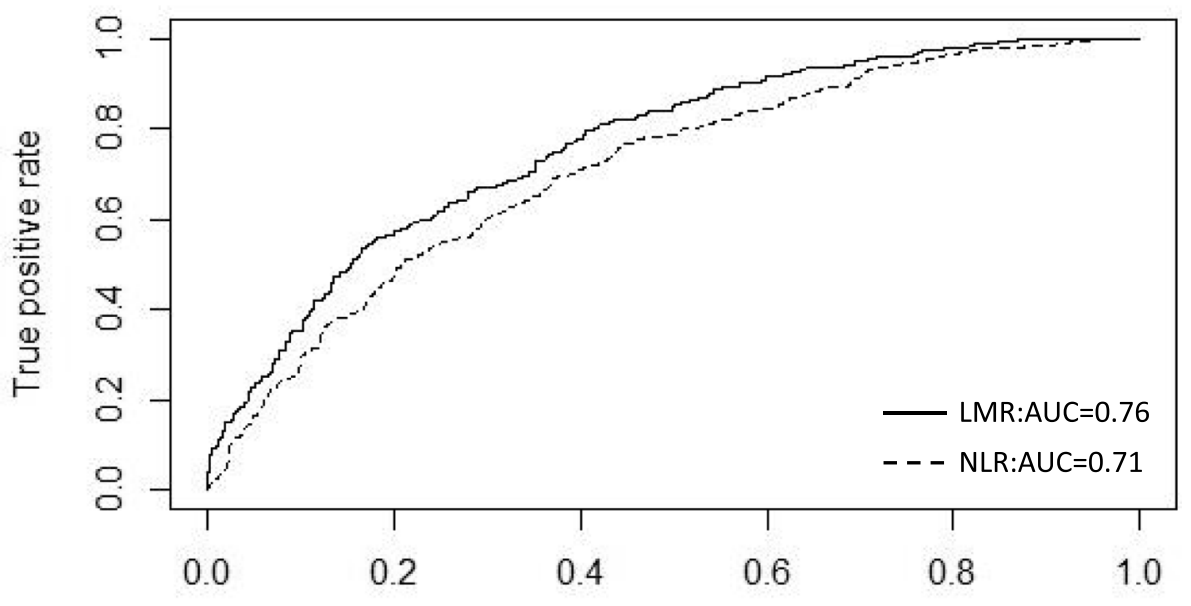

False positive rate

Fig. 2 The ROC curves for LMR and NLR to differentiate pneumonia from URTI on the validation set 


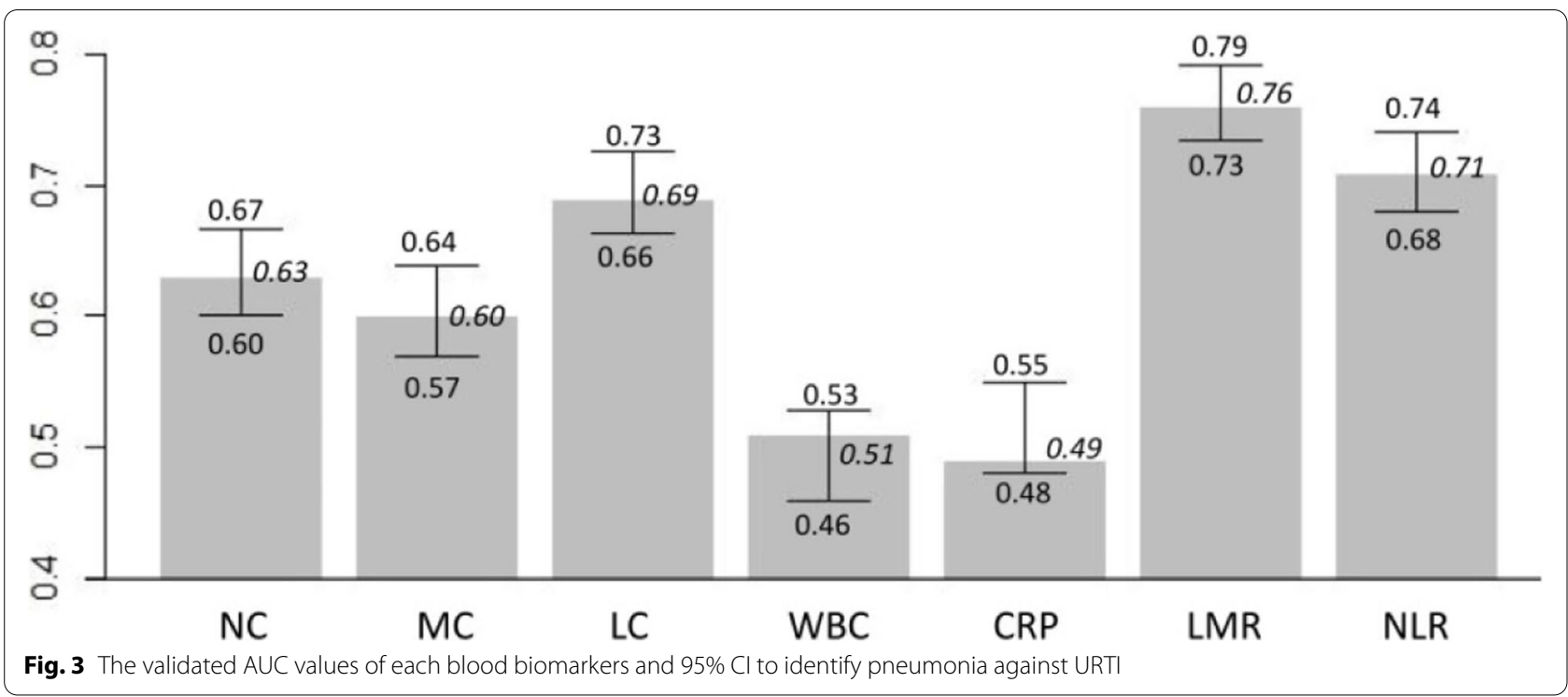

CI: 0.74-0.80) and 0.71 (95\% CI: 0.67-0.74) in differentiating bacterial pneumonia against URTI (see Supplementary Fig. 1).

Recognized as the two strongest predictors of pneumonia in children, LMR was found to increase the risk of viral, bacterial and combined pneumonia when considering URTI as reference, and it attained ORs of 1.24, 1.26 and 1.27 respectively in our study. In contrast, NLR decreased the risk of three different types of pneumonia, with ORs of $0.23,0.73$ and 0.67 respectively (see Supplementary Table 4).

Since LMR and NLR were validated as valuable predictors of pneumonia, reaching relatively high classification accuracy individually, we also investigated whether the integration of the two indicators with other important clinical symptoms could further construct a multivariate screening model with improved accuracy. The results showed that, when combining age, clinical symptoms (i.e., fever, cough and rhinorrhea) together with LMR and NLR, the screening model reached an AUC of 0.84 (95\% CI: 0.82-0.87) for discriminating the overall pneumonia from URTI, better than the model considering LMR and NLR (AUC $=0.76[0.73-0.78]$ ) only or the model using clinical symptoms and age $(\mathrm{AUC}=0.81[0.78-0.83])$ alone (see Fig. 4). It is worth noting that in the subgroup analysis for identifying viral pneumonia from URTI, the integrated model reached an AUC value as high as 0.95 (95\% CI: 0.93-0.97), outperformed than the other two compared models. As for identifying bacterial pneumonia from URTI, the integrated model reached an AUC of 0.86 (0.83-0.88). We further investigated whether the integration of LMR and NLR with other important clinical symptoms could construct a multivariate screening model to differentiate bacterial pneumonia from viral pneumonia. The results showed that, when combining age, clinical symptoms (i.e., fever, cough and rhinorrhea) together with LMR and NLR, the integrated screening model reached an AUC of 0.83 (0.80-0.87), still slightly better than the model considering LMR and NLR (AUC $=0.75)$ only and the model using clinical symptoms and age $(\mathrm{AUC}=0.82)$ alone. The details were all carefully introduced in Supplementary Fig. 2.

Besides, several studies have illustrated that CRP could be used to assist pneumonia diagnosis [22], thus we consequently combined CRP, age and clinical signs to build a new multivariate screening model and compared its discriminating ability with our integrated model using LMR and NLR. The results showed that, although the CRP-involved multivariate model attained a relatively high validated AUC value of 0.81 (95\% CI: 0.79-0.84) in distinguishing the overall pneumonia from URTI, it was still lower than our multivariate screening model recruiting LMR and NLR. The relatively high performance of the CRP-involved model was indeed attributed to the good screening ability of included clinical signs. Subgroup (viral and bacterial) analysis also indicated similar result that our integrated model achieved better discriminating ability than the CRP-involved model, which was carefully demonstrated in Supplementary Fig. 3.

Considering that age profoundly affects incidence and development of most pediatric diseases, we also explored the influence of age on the model's performance and revealed the model's capability across various age stratifications. As the results showed, the multivariate screening model attained better performance in all age groups, compared to the other two models, with its best performance at 1-3 (AUC:0.83) and over 7 (AUC:0.87) age 


\section{Pneumonia}

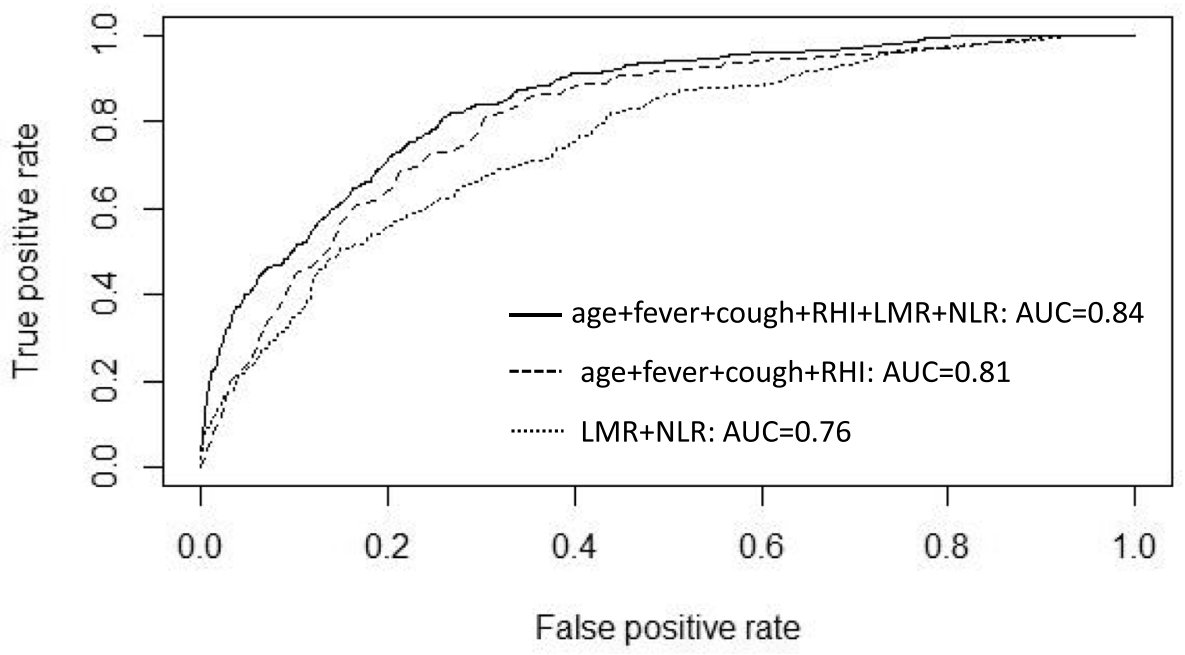

Fig. 4 The ROC curve of three different models to discriminate pneumonia against URTI

groups. After calculating the PPVs, sensitivity and specificity for the identified high-risk category within various age stratifications, it was found that the built multivariate screening model reached steady and relatively high PPVs across all age groups, with all values $>80 \%$. In terms of sensitivity and specificity, the built model attained the highest sensitivity of $62.26 \%$ in the $1-3$ age group, and best specificity of $23.53 \%$ in the $0-1$ age group (Supplementary Table 5). It was worth noting that in children of $0-3$ years old, the model only integrating LMR and NLR performed worse than the other two models, only attaining AUC values of 0.68 and 0.64 at the age of $0-1$ and 1-3years old respectively. With the growing age, the model only integrating LMR and NLR significantly outperformed the model with clinical symptoms alone, by attaining AUCs of 0.81 in children over 7 years old. More details could be found in Fig. 5 .

Given this multivariate screening model, we calculated the risk scores for each individual in either training or validation sets. Then, by using the cut off values (i.e., the 25 and $75 \%$ quantiles) generated from training set, we classified subjects in the validation set into highrisk categories if their risk scores $\geq 0.763$, and identified individuals as medium- or low-risk ones if their risk scores ranged between 0.763 and 0.213 , or $\leq 0.213$, respectively. Finally, with a total of 1043 individuals in the validation cohort, the multivariate screening model classified $24.07 \%(251 / 1043)$ of them into the high-risk category, with a PPV as high as $87.25 \%$, whereas it identified $52.16 \%(544 / 1043)$ and $23.78 \%(248 / 1043)$ of individuals into the medium and low risk groups of the overall pneumonia, with PPVs of 49.26 and 9.2\%, respectively
(Table 2). In addition, following the same rationale of using the 25 and $75 \%$ quantiles generated from training set as cut-offs, we also classified individuals of the validation set into three risk categories under both the model considering clinical symptoms alone and the model only integrating LMR and NLR respectively. It was worth noting that these two models only reached PPVs of 74.82 and $77.13 \%$ in the high-risk category respectively, lower than that of the multivariate screening model. Meanwhile, the model considering signs alone attained the sensitivity as high as $61.76 \%$ in the high-risk group, followed by our multivariate screening model and the model only considering LMR and NLR, with sensitivities of 42.94 and 33.73\% respectively (Supplementary Table 6 and Supplementary Table 7).

\section{Discussion}

In this study, we constructed a univariate screening model considering blood biomarkers only and a multivariate screening model that integrated both blood biomarkers and clinical symptoms to identify pneumonia from URTI in children. As validated, the single-variate screening model of LMR and NLR attained AUCs of 0.76 and 0.71 respectively, outperformed other biomarkers (AUC: 0.49-0.69) in regular blood testing on screening accuracy. After further integrating LMR and NLR with three common clinical symptoms of fever, cough and rhinorrhea, the multivariate screening model obtained an increased discriminative ability, reached an AUC of 0.84 for distinguishing pneumonia from URTI, better than using clinical symptoms only $(\mathrm{AUC}=0.81)$. According to the risk scores of the multivariate model, we stratified 


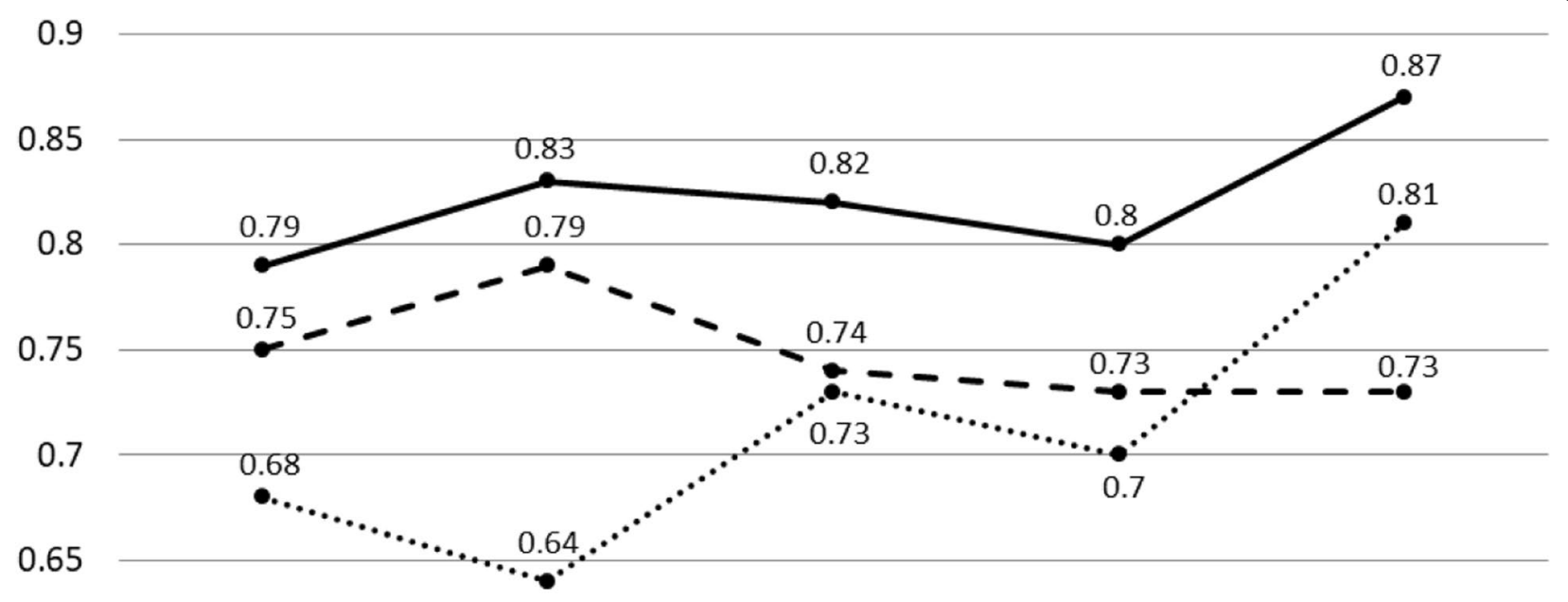

\section{6}

\subsection{5}

\section{5}

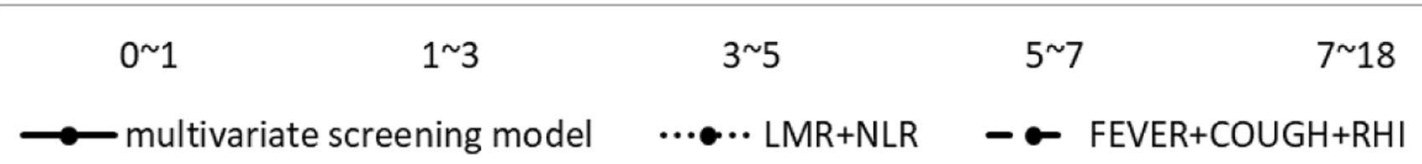

Fig. 5 The performance of three models at different age stratifications

Table 2 The performance of multivariate screening model for overall pneumonia in the cohort

\begin{tabular}{lllll}
\hline & High risk & Medium risk & Low risk & Total \\
\hline Total & 251 & 544 & 248 & 1043 \\
Case & 219 & 268 & 23 & 510 \\
PPV & $87.25 \%$ & $49.26 \%$ & $9.27 \%$ & $48.90 \%$ \\
Sensitivity & $42.94 \%$ & $52.55 \%$ & $4.51 \%$ & \\
Specificity & $6.00 \%$ & $51.78 \%$ & $42.21 \%$ & \\
\hline
\end{tabular}

individuals into three distinct pneumonia-risk categories (high, medium and low), and found that, $87.25 \%$ of the pneumonia children were successfully identified by the model as high risk. On the contrary, only $9.27 \%$ of the cases were subject to low-risk group.

As an easy and inexpensive routine examination technique, complete blood counts can provides information about WBC, neutrophil, C-reactive protein, monocyte and lymphocyte, therefore, the ratio of neutrophils to lymphocytes (NLR) can be easily calculated [23-25]. Our current study adds to the value of the NLR by showing that this marker is of interest in distinguishing pneumonia from URTI in children, we found that the discriminatory capacity of NLR outweighed predictive values of traditional biomarkers such as CRP, which has been identified elsewhere as a good indicator of CAP risk (community-acquired pneumonia risk) [22]. It has also been reported that, compared with healthy people, NLR was significantly increased in pneumonia, indicating that it can be used as predictors for the presence of pneumonia $[26,27]$. A previous study suggested NLR as an indicator to identify adults with pneumonia, which attained an AUC of 0.938 [28], higher than that of our study. The difference in performance may partly due to the heterogeneity of the study population, for they aimed to capture pneumonia cases from healthy adults while we distinguished pneumonia from URTI in children. However, the underlying cause of lower NLR level in pneumonia group than that of URTI has not been yet clear. Lower NLR was defined as increased lymphocyte counts or decreased neutrophils count. Lymphocytes are present in the lung and have been shown to play a role in several lung diseases (pneumonia, asthma, chronic obstructive pulmonary disease and so on) in both humans and mice $[29,30]$. More specifically, lymphocytes are recruited to the lung in response to pulmonary infections (Aspergillus 
fumigatus, Klebsiella pneumonia) [31]. It has been reported that increased lymphocytosis is associated with pulmonary hypertension, pneumonia, and death [32]. Fabienne Venet et al. observed the different lymphocyte subpopulations present and/or recruited to the lung in the development of acute lung injury (ALI), where ALI was mainly caused by pneumonia. Mice data suggested that, during the ALI, the recruiting of CD4+ T lymphocytes to the lung is partly activated by the increased level of IL-16 produced in ECs (lung endothelial cells), whereas the recruiting of neutrophils is inhibited by the increased IL-10 level during these process [33], which supported our observed lower level of NLR in pneumonia children.

Several recent studies suggest that the LMR is an economical, readily available and reproducible test for predicting clinical outcomes of patients with solid tumors and hematological malignancy, including nasopharyngeal carcinoma, colorectal cancer and lymphoma and so on [34-36]. Moreover, Merekoulias et al. found that in $90 \%$ of patients who had influenza virus, lymphopenia and/ or monocytosis, and LMR could be used as a time-saving and cost-effective screening test for influenza virus infection [37]. Our study proved the potential utility of this infection marker in children to discriminate pneumonia from URTI. It's worth noting that the discriminatory capacity of the LMR outweighed predictive values of NLR in identifying the overall pneumonia as well as bacterial pneumonia, but lower than that of NLR in the identification of viral pneumonia. The difference may partly due to the relatively small number (460) of children affected by viral pneumonia enrolled in our study, compared with 2088 cases with bacterial pneumonia. Elevated LMR was defined as decreased monocyte or increased lymphocyte counts. However, compared to URTI, the relationship between higher levels of LMR in pneumonia is not yet clear. In acute lung inflammation, blood monocytes migrate into the lung parenchyma and bronchoalveolar space, where they differentiate into "monocyte-derived" alveolar macrophages (Mo-AMs) and orchestrate a proinflammatory and profibrotic response, leading to the decreased level of monocyte count in the blood [38-41]. In the lung, the newly arrived monocytes can enhance microbicidal activities by producing TNF and nitric oxide synthases [42, 43]. Moreover, Yong found that monocyte chemoattractant protein-1 increases in the serum of immunocompetent patients with CAP [44], which may lead to the reduced concentration of monocyte in the blood, as well as the decreased level of LMR comparing to URTI.

In terms of application, we hope this easy-to-implement pneumonia primary screening tool could be utilized in community health centers or resource-poor primary care settings in several ways. First, it may be helpful in triage procedures. For instance, when children come to primary health care centers, their vital signs and blood routine value would be entered into the model and generate a predicted pneumonia risk. These predicted results could help to prioritize which children should be sent for chest radiography examination, and thus would help to ensure that medical resources and equipment is dedicated to children with the highest needs. By triaging patients more effectively, corresponding radiation hazards can be eliminated, and avoidable medical expenses and family burdens caused by excessive medical treatment will ultimately be reduced. Another potential application of this screening tool is to assist primary physicians to improve the accuracy of diagnosis. While formulating a differential diagnosis, community physicians often draw on their past experience and may not have extensive expertise for patients presenting with similar signs, so misdiagnosis may be a distinct possibility in these cases. Using this screening tool, community physicians can use the risk derived from the model to help improve the accuracy of his differential diagnosis, thereby reducing the bias of individual physicians.

Our study has several limitations. First, the bacterial pneumonia and viral pneumonia was not balanced in our data, which might cause bias in terms of risk assessment and performance evaluation. Second, in this study, although pediatricians made a comprehensive diagnosis of URTI based on clinical signs, blood routine tests, and possible chest X-ray results, case of bronchitis, bronchiolitis or wheezing, which were true LRTI, may still be potentially misclassified as URTI, however, such misclassification rate should be low. Third, this study only included three common signs (fever, cough and rhinorrhea) into the screening model, while some important clinical symptoms such as tachypnea were not recorded as structured data in the hospital information system and thus could not be accurately and directly extracted from the big data platform. Since such symptoms are potentially valuable indicators of pneumonia, the missing information may reduce the accuracy of the developed screening tool. Finally, in this study, the bacterial or viral infections were not labeled for upper respiratory tract infection patients in the hospital information system, thus it is hoped that the predictive value and the underlying mechanism of the two indicators, NLR and LMR, in identifying different sub-types of pneumonia and URTI could be further elucidated.

\section{Conclusion}

In conclusion, we have constructed and validated screening models to assess pneumonia risk in children. The multivariate screening model achieved a 0.84 predictive accuracy in validation cohorts, and successfully stratified 
patients into three distinct pneumonia risk categories. We hope that the constructed pneumonia screening tool for children could be applied and evaluated further in primary health center to explore its utility and cost effectiveness, and ultimately facilitate clinical decision making for children's pneumonia diagnosis at the community level.

\section{Abbreviations}

URTI: Upper Respiratory Tract Infection; LMR: Lymphocyte-To-Monocyte Ratio; NLR: Neutrophil-To-Lymphocyte Ratio; STARD: Standard for Reporting of Diagnostic Accuracy Studies; NC: Neutrophils Cell; MC: Monocytes Cell; LC: Lymphocyte Cell; WBC: White Blood Cell Count; CRP: C-Reactive protein; PCR: Polymerase chain reaction; RHI: Rhinorrhea; GGO: Ground-glass opacity; RSV: Respiratory Syncytial Virus; ADVDNA: Adenovirus Nucleic Acid; FluA-Ag: Influenza A virus Antigen; PPVs: Positive predictive values.

\section{Supplementary Information}

The online version contains supplementary material available at https://doi. org/10.1186/s12887-021-03018-y.

Additional file 1 Supplementary Figure 1. The ROC curve for LMR and NLR to differentiate two different types of pneumonia against URTI. The figure shows the subgroup analysis of LMR and NLR in distinguishing URTI from viral or bacterial pneumonia.

Additional file $\mathbf{2}$ Supplementary Figure 2. The ROC curve of three models to identify two different types of pneumonia against URTI, as well as to differentiate bacterial pneumonia from viral pneumonia. The figure shows the subgroup analysis of three models to identify two different types of pneumonia against URTI, as well as to differentiate bacterial pneumonia from viral pneumonia.

Additional file 3 Supplementary Figure 3. The ROC curve of combing age, fever, cough, $\mathrm{RHI}$ and CRP to identify two different types of pneumonia against URTI. The figure shows the model considering age, fever, cough, RHI and CRP to identify URTI against overall pneumonia, as well as two different types of pneumonia.

Additional file 4 Supplementary Table 1. STARD checklist. STARD checklist contains a list of essential items to make sure the report of a diagnostic accuracy study contains the necessary information.

Additional file 5 Supplementary Table 2. Diagnostic criteria for URTI and pneumonia. This file contains a table of diagnostic criteria and procedures for URTI and pneumonia, as well as a paragraph to fully explain it.

Additional file 6 Supplementary Table 3. AUC values for each blood biomarkers to differentiate pneumonia, viral pneumonia and bacterial pneumonia from URTI. This table shows the AUC values for each blood biomarkers in identifying pneumonia, viral pneumonia and bacterial pneumonia against URTI.

Additional file 7 Supplementary Table 4. Correlation between LMR, NLR and three different types of pneumonia. This table shows the relationship between LMR, NLR and pneumonia.

Additional file $\mathbf{8}$ Supplementary Table 5. The distribution of high-risk children across the different age groups, identified by the built multivariate screening model. This table shows PPVs, sensitivity and specificity of high-risk children identified by the built multivariate screening model across various age stratifications.

Additional file 9 Supplementary Table 6. The performance of the model considering clinical signs alone for overall pneumonia in the cohort. This table shows PPVs, sensitivity and specificity of the model considering clinical signs alone for overall pneumonia.

Additional file 10 Supplementary Table 7. The performance of the model only considering LMR and NLR for overall pneumonia in the cohort. This table shows PPVs, sensitivity and specificity of the model only considering LMR and NLR for overall pneumonia.

\section{Acknowledgements}

The authors would like to thank all the staff of Zhejiang Provincial People's Hospital for diagnosis and collection of blood samples.

\section{Authors' contributions}

Jinghua Wu contributed to the data analysis, manuscript writing, review and editing. Xu Wang and Mingqi Zhou participated in data curation and analysis. Guo-Bo Chen and Jing Du oversaw data analytical methods. Ying Wang and Chengyin Ye contributed to the study design and conception. All authors have read and agreed to the published version of the manuscript.

\section{Funding}

This study has been funded by the National Natural Science Foundation of China [grant number:81402762 to CYY, 81971172 to YW, 31771392 to GBC), Project of Engineering Research Center of Ministry of Education of Mobile Health Management [grant number: PD2015304], Zhejiang Provisional People's Hospital Research Startup Fund [grant number: ZRY2018A004], The Key Research and Development Program of Zhejiang Province [grant number: WKJZJ-1914], Zhejiang Natural Science Foundation [grant number: LY18C09004], Medical and Health Science and Technology Project of Zhejiang Province [grant number:2018KY003, 2018KY816), Zhejiang Public Welfare Technology Research Program [grant number: LGF18H090009], and Analysis and Testing Technology Project of Zhejiang Provincial Department of Science and Technology [grant number:2018C37078]. The funding bodies were not involved in any process of study design, data collection, analysis, or manuscript writing.

\section{Availability of data and materials}

The datasets used during the current study are available from the corresponding author on reasonable request.

\section{Declarations}

\section{Ethics approval and consent to participate}

This study was approved by ethics committee of Zhejiang Provincial People's Hospital (No. 2021QT222), and was exempt from informed consent as shown by the IRB approval letter.

\section{Consent for publication}

Not applicable.

\section{Competing interests}

The authors declare that they have no known competing financial interests or personal relationships that could have appeared to influence the work reported in this paper.

\section{Author details}

${ }^{1}$ Department of health management, Hangzhou Normal University, Zhejiang 310000, Hangzhou, China. ${ }^{2}$ Engineering Research Center of Mobile Health Management System, Ministry of Education, Hangzhou Normal University, Zhejiang 310000, Hangzhou, China. ${ }^{3}$ Department of Laboratory Medicine, Zhejiang Provincial People's Hospital, People's Hospital of Hangzhou Medical College, Zhejiang 310014, Hangzhou, China. ${ }^{4}$ School of Laboratory Medicine and Life Science, Wenzhou Medical University, Wenzhou, Zhejiang 325035, China. ${ }^{5}$ Key Laboratory of Endocrine Gland Diseases of Zhejiang Province, Zhejiang 310000, Hangzhou, China. ${ }^{6}$ Phase I Clinical Research Center, Zhejiang Provincial People's Hospital, Affiliated People's Hospital, Hangzhou Medical College, Zhejiang 310000, Hangzhou, China.

Received: 22 July 2021 Accepted: 17 November 2021

Published online: 03 December 2021

\section{References}

1. Liu L, Oza S, Hogan D, Chu Y, Perin J, Zhu J, et al. Global, regional, and national causes of under-5 mortality in 2000-15: an updated systematic analysis with implications for the Sustainable Development Goals. Lancet (London, England). 2016:388:3027-35.

2. Cooke G, Valenti L, Glasziou P, Britt H. Common general practice presentations and publication frequency. Aust Fam Physician. 2013;42:65-8. 
3. Gray D, Willemse L, Visagie A, Czövek D, Nduru P, Vanker A, et al. Determinants of early-life lung function in African infants. Thorax. 2016;72:445-50.

4. Svanes C, Sunyer J, Plana E, Dharmage S, Heinrich J, Jarvis D, et al. Early life origins of chronic obstructive pulmonary disease. Thorax. 2010;65:14-20.

5. Chan JYC, Stern DA, Guerra S, Wright AL, Morgan WJ, Martinez FD. Pneumonia in childhood and impaired lung function in adults: a longitudinal study. Pediatrics. 2015;135:607-16.

6. Martinez FD. Early-life origins of chronic obstructive pulmonary disease. N Engl J Med. 2016;375:871-8.

7. Cherian T, Mulholland EK, Carlin JB, Ostensen H, Amin R, de Campo M, et al. Standardized interpretation of paediatric chest radiographs for the diagnosis of pneumonia in epidemiological studies. Bull World Health Organ. 2005:83:353-9.

8. van Vugt SF, Broekhuizen BDL, Lammens C, Zuithoff NPA, de Jong PA, Coenen $\mathrm{S}$, et al. Use of serum $\mathrm{C}$ reactive protein and procalcitonin concentrations in addition to symptoms and signs to predict pneumonia in patients presenting to primary care with acute cough: diagnostic study. BMJ. 2013;346:f2450

9. Leung AKC, Wong AHC, Hon KL. Community-Acquired Pneumonia in Children. Recent Pat Inflamm Allergy Drug Discov. 2018;12:136-44.

10. Jain N, Lodha R, Kabra SK. Upper respiratory tract infections. Indian J Pediatr. 2001:68:1135-8.

11. Jain S, Williams DJ, Arnold SR, Ampofo K, Bramley AM, Reed C, et al. Community-acquired pneumonia requiring hospitalization among U.S. children. N Engl J Med. 2015;372:835-45.

12. Anand N, Kollef MH. The alphabet soup of pneumonia: CAP, HAP, HCAP, NHAP, and VAP. Semin Respir Crit Care Med. 2009;30:3-9.

13. Jain S, SelfWH, Wunderink RG, Fakhran S, Balk R, Bramley AM, et al. Community-acquired pneumonia requiring hospitalization among U.S. adults. N Engl J Med. 2015;373:415-27.

14. Mizgerd JP. Inflammation and pneumonia: why are some more susceptible than others? Clin Chest Med. 2018;39:669-76.

15. Quinton $\sqcup$, Walkey AJ, Mizgerd JP. Integrative physiology of pneumonia. Physiol Rev. 2018;98:1417-64.

16. Cline MJ, Lehrer RI, Territo MC, Golde DW. UCLA conference. Monocytes and macrophages: functions and diseases. Ann Intern Med. 1978;88:78-88.

17. Gu X, Sun S, Gao X-S, Xiong W, Qin S, Qi X, et al. Prognostic value of platelet to lymphocyte ratio in non-small cell lung cancer: evidence from 3,430 patients. Sci Rep. 2016;6:23893.

18. Kolaczkowska E, Kubes P. Neutrophil recruitment and function in health and inflammation. Nat Rev Immunol. 2013;13:159-75.

19. Köse N, Ylldıım T, Akın F, Yıldıım SE, Altun I. Prognostic role of NLR, PLR, and LMR in patients with pulmonary embolism. Bosn J basic Med Sci. 2020:20:248-53.

20. Yevich S, Gaspar N, Tselikas L, Brugières L, Pacquement H, Schleiermacher $\mathrm{G}$, et al. Percutaneous computed tomography-guided thermal ablation of pulmonary osteosarcoma metastases in children. Ann Surg Oncol. 2016:23:1380-6.

21. Cohen JF, Korevaar DA, Altman DG, Bruns DE, Gatsonis CA, Hooft L, et al. STARD 2015 guidelines for reporting diagnostic accuracy studies: explanation and elaboration. BMJ Open. 2016;6:1-17.

22. Alcoba G, Keitel K, Maspoli V, Lacroix L, Manzano S, Gehri M, et al. A threestep diagnosis of pediatric pneumonia at the emergency department using clinical predictors, C-reactive protein, and pneumococcal PCR. Eur J Pediatr. 2017:176:815-24.

23. Özdin S, Sarisoy G, Böke Ö. A comparison of the neutrophil-lymphocyte, platelet-lymphocyte and monocyte-lymphocyte ratios in schizophrenia and bipolar disorder patients - a retrospective file review. Nord J Psychiatry. 2017;71:509-12.

24. Li Z, Hong N, Robertson M, Wang C, Jiang G. Preoperative red cell distribution width and neutrophil-to-lymphocyte ratio predict survival in patients with epithelial ovarian cancer. Sci Rep. 2017;7:43001.

25. Feng F, Sun L, Zheng G, Liu S, Liu Z, Xu G, et al. Low lymphocyte-to-white blood cell ratio and high monocyte-to-white blood cell ratio predict poor prognosis in gastric cancer. Oncotarget. 2017;8:5281-91.

26. Pantzaris N-D, Platanaki C, Pierrako C, Karamouzos V, Velissaris D. Neutrophilto-lymphocyte ratio relation to Sepsis severity scores and inflammatory biomarkers in patients with community-acquired pneumonia: a case series. JTransl Intern Med. 2018;6:43-6.

27. Kartal O, Kartal AT. Value of neutrophil to lymphocyte and platelet to lymphocyte ratios in pneumonia. Bratisl Lek Listy. 2017;118:513-6.
28. Huang Y, Liu A, Liang L, Jiang J, Luo H, Deng W, et al. Diagnostic value of blood parameters for community-acquired pneumonia. Int Immunopharmacol. 2018:64:10-5.

29. Holt PG, Strickland DH, Wikström ME, Jahnsen FL. Regulation of immunological homeostasis in the respiratory tract. Nat Rev Immunol. 2008;8:142-52.

30. Greenberger PA. 7. Immunologic lung disease. J Allergy Clin Immunol. 2008;121(2 Suppl):S393-7 quiz S418.

31. Moore TA, Moore BB, Newstead MW, Standiford TJ. Gamma delta-T cells are critical for survival and early proinflammatory cytokine gene expression during murine Klebsiella pneumonia. J Immunol. 2000;165:2643-50.

32. Funaki T, Miyairi I. Lymphocytosis in a baby with pertussis. Lancet Infect Dis. 2015;15:130.

33. Venet F, Chung C-S, Huang X, Lomas-Neira J, Chen Y, Ayala A. Lymphocytes in the development of lung inflammation: a role for regulatory CD4+ T cells in indirect pulmonary lung injury. J Immunol. 2009;183:3472-80.

34. Belotti A, Doni E, Bolis S, Rossini F, Casaroli I, Pezzatti S, et al. Peripheral blood lymphocyte/monocyte ratio predicts outcome in follicular lymphoma and in diffuse large B-cell lymphoma patients in the rituximab era. Clin Lymphoma Myeloma Leuk. 2015;15:208-13.

35. Lin G-N, Peng J-W, Liu D-Y, Xiao J-J, Chen Y-Q, Chen X-Q. Increased lymphocyte to monocyte ratio is associated with better prognosis in patients with newly diagnosed metastatic nasopharyngeal carcinoma receiving chemotherapy. Tumour Biol. 2014;35:10849-54.

36. Paik KY, Lee IK, Lee YS, Sung NY, Kwon TS. Clinical implications of systemic inflammatory response markers as independent prognostic factors in colorectal cancer patients. Cancer Res Treat. 2014:46:65-73.

37. Merekoulias G, Alexopoulos EC, Belezos T, Panagiotopoulou E, Jelastopulu DME. Lymphocyte to monocyte ratio as a screening tool for influenza. Plos Curr. 2010:2:RRN1154.

38. Atochina-Vasserman EN, Bates SR, Zhang P, Abramova H, Zhang Z, Gonzales $L$, et al. Early alveolar epithelial dysfunction promotes lung inflammation in a mouse model of Hermansky-Pudlak syndrome. Am J Respir Crit Care Med. 2011:184:449-58.

39. Gibbons MA, MacKinnon AC, Ramachandran P, Dhaliwal K, Duffin R, Phythian-Adams AT, et al. Ly6Chi monocytes direct alternatively activated profibrotic macrophage regulation of lung fibrosis. Am J Respir Crit Care Med. 2011;184:569-81.

40. Janssen WJ, Barthel L, Muldrow A, Oberley-Deegan RE, Kearns MT, Jakubzick

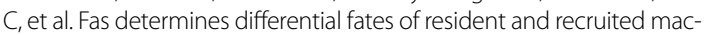
rophages during resolution of acute lung injury. Am J Respir Crit Care Med. 2011;184:547-60.

41. Misharin AV, Morales-Nebreda L, Reyfman PA, Cuda CM, Walter JM, McQuattie-Pimentel AC, et al. Monocyte-derived alveolar macrophages drive lung fibrosis and persist in the lung over the life span. J Exp Med. 2017:214:2387-404

42. Tsuchimoto Y, Asai A, Tsuda Y, Ito I, Nishiguchi T, Garcia MC, et al. M2b monocytes provoke bacterial pneumonia and gut Bacteria-associated Sepsis in alcoholics. J Immunol. 2015;195:5169-77.

43. Casson CN, Doerner JL, Copenhaver AM, Ramirez J, Holmgren AM, Boyer MA, et al. Neutrophils and Ly6Chi monocytes collaborate in generating an optimal cytokine response that protects against pulmonary legionella pneumophila infection. PLoS Pathog. 2017;13:e1006309.

44. YongKK, Chang JH, Chien MH, Tsao SM, Yu MC, Bai KJ, et al. Plasma monocytechemoattractant protein-1 level as a predictor of the severity ofcommunity-acquired pneumonia. Int J Mol Sci. 2016;17:179

\section{Publisher's Note}

Springer Nature remains neutral with regard to jurisdictional claims in published maps and institutional affiliations. 